\title{
Methodology to determine residence time distribution and small scale phenomena in settling tanks
}

\author{
T. Karches \& K. Buzas \\ Department of Sanitary and Environmental Engineering, \\ Budapest University of Technology and Economics, Hungary
}

\begin{abstract}
Hydraulic residence time is a crucial parameter of any wastewater treatment tank planning, operation and optimisation. Calculation of the residence time distribution at every point within the tank gives information about the deadzones and short circuits and well-operating zones. Using the advective-diffusive transport equation supplemented with a scalar source term local mean age (LMA) can be detected if the flow field is given. Combining LMA with a tracer study the exact residence time can be determined. This method supports problem detection in a small wastewater treatment plant ( 2 dead zones and one shortcircuiting path were detected) and also gave us better understanding of the operation of settling tanks.
\end{abstract}

Keywords: CFD, LMA, residence time, settling tank design.

\section{Introduction}

Computational fluid dynamics (CFD) in wastewater treatment processes has a significant role in recent years. Several studies demonstrate that CFD is an effective tool in design (Greenfield [8]), optimization (Bratchley et al. [3]) and operation (Brouckaert and Buckley [4]) Furthermore CFD studies revealed that with an extensive knowledge of fluid flow not only the hydraulic performance of e.g. an oxidation ditch could be improved (Yang et al. [17]) but we could have a closer look into the biological processes, too (Bartrand et al. [2]) For a few decades researchers used to focus only on the biology and chemistry in wastewater treatment, and hydraulics was mostly neglected. 
According to ATV-A131 standard [1] the settling tanks have been handled by black box model, of which key parameter is hydraulic residence time. Most cases use a constant residence time - despite the fact that there is a distribution of residence time - calculated by $\mathrm{t}=\mathrm{V} / \mathrm{Q}$, where $\mathrm{V}$ is the volume of the settling tank in $\mathrm{m}^{3}$ and $\mathrm{Q}$ is the influent wastewater volume flux in $\mathrm{m}^{3} / \mathrm{s}$.

Residence time distribution (RTD) can be calculated using tracer study both experimentally and numerically (Gentric [7]). The field measurement based on the idea that a certain amount of $\mathrm{LiCl}$ is emitted to the wastewater tank and the concentration as the response function was measured as the function of time at the outlet. $\mathrm{LiCl}$ is a conservative material that never constituent of raw wastewater. The measured concentration data show how fast the particles go through the system, therefore this is useful in determining whether there are short -circuits or dead-zones and gives information how the wastewater tanks operate. If we know the flow, velocity and pressure fields, furthermore turbulence parameters, the tracer study can also be carried out numerically. If the tracer is injected at a short time interval, small concentrations will appear at the outflow where the rounding error's order of magnitude is close to the detected concentration. Eliminating this uncertainty the tracer is emitted to the inflow continuously and the response function is a cumulative function of concentration, which shows how fast the original fluid replaced by the tracer.

Numerical hydrodynamics should be completed with multiphase modelling in order to describe the behaviour of wastewater (and components) in the treatment process. Many efforts have been made, generally using particle tracking method (Saul and Stevin [14]), but when there is a strong coupling between fluid flow and the secondary phase, Eulerian-Eulerian multiphase model is more suitable (Ta et al. [16]).

Other approach to detect the spatial distribution of the fluid particles' age is the Local Mean Age (LMA) theory. LMA is used basically in ventilation techniques to describe mixing phenomenon of the air (Simons et al. [15]), but this method can be applied to water flow, too. Using the convection-diffusion scalar transport equation with zero-order kinetics the mean age of water can be calculated at each point in the tank and the computation time is less than in the RTD method. Furthermore, with LMA method reveal the problematic zones in the wastewater tanks and the geometry could be reconstruct or new boundary conditions could be set.

The theory section describes the mathematical background of this study. The results section presents a case study, where RTD and LMA methods were applied in a small wastewater plant in Sarisap, Hungary.

\section{Theory of numerical methods}

For the description of the fluid flow the conservation equations for mass and for the momentum has to be solved (Foias et al. [9]). 
Conservation of mass

$$
\frac{\partial \rho}{\partial t}+\nabla \cdot(\rho \vec{v})=0
$$

Conservation of momentum

$$
\frac{\partial(\rho \vec{v})}{\partial t}+\nabla \cdot(\rho \vec{v} \vec{v})=-\nabla p+\nabla \cdot(\overline{\bar{\tau}})+\rho \vec{g}+\vec{F}
$$

where $p$ is the static pressure, $\rho \vec{g}$ is gravitational body force, $\vec{F}$ is external body force and $\overline{\bar{\tau}}$ is stress tensor, what can be written in form of:

$$
\overline{\bar{\tau}}=\mu\left[\left(\nabla \vec{v}+\nabla \vec{v}^{T}\right)-\frac{2}{3} \nabla \cdot \vec{v} I\right],
$$

where $\mu$ is molecular viscosity and $I$ is the unit tensor.

Closure for the partial differential equation system is the k- $\varepsilon$ turbulence model, where one equation is solved for the turbulence kinetic energy (k), see in eqn (4) and one for the turbulent dissipation $(\varepsilon)$, see in eqn (5). This model assumes isotropic turbulence (Launder and Spalding [12]).

$$
\begin{gathered}
\frac{\partial(\rho k)}{\partial t}+\frac{\partial}{\partial x_{j}}\left(\rho k u_{j}\right)=\frac{\partial}{\partial x_{j}}\left[\left(\mu+\frac{\mu_{t}}{\sigma_{k}}\right) \frac{\partial k}{\partial x_{j}}\right]+G_{k}+G_{b}-\rho \varepsilon \\
\frac{\partial(\rho \varepsilon)}{\partial t}+\frac{\partial}{\partial x_{j}}\left(\rho \varepsilon u_{j}\right)=\frac{\partial}{\partial x_{j}}\left[\left(\mu+\frac{\mu_{t}}{\sigma_{\varepsilon}}\right) \frac{\partial \varepsilon}{\partial x_{j}}\right] \\
-\rho C_{2} \frac{\varepsilon^{2}}{k+\sqrt{v \varepsilon}}+C_{1 \varepsilon} \frac{\varepsilon}{k} C_{3 \varepsilon} G_{b}
\end{gathered}
$$

where $G_{k}$ is generation of the turbulent kinetic energy due to the mean velocity tensor and calculated as:

$$
G_{k}=\mu_{t} S^{2}
$$

$S$ is the deformation tensor

$$
S \equiv \sqrt{2 S_{i j} S_{i j}}
$$


$\mu_{t}$ is the eddy viscosity

$$
\mu_{t}=\rho C_{\mu} \frac{k^{2}}{\varepsilon}
$$

Here $C_{\mu}$ is a function of the mean strain and rotation rates, the angular velocity of the system rotation and the turbulence fields. $G_{b}$ is generation of turbulent kinetic energy due to the buoyancy. Model constants are:

$$
\mathrm{C}_{1 \varepsilon}=1.44, \mathrm{C}_{2 \varepsilon}=1.9, \sigma_{\mathrm{k}}=1, \sigma_{\varepsilon}=1.2
$$

For the LMA calculation an additional convection-diffusion scalar transport equation needs to be solved:

$$
\frac{\partial \rho \varphi}{\partial t}+\nabla \cdot(\rho \vec{v} \varphi-\Gamma \nabla \varphi)=S
$$

where $\rho$ is the fluid density, $\varphi$ is the local mean age of the fluid $\mathrm{v}$ is the fluid velocity, $\Gamma$ is the diffusion coefficient, $S$ is the source term of the scalar. $S$ is taken as equal to 1 and for the diffusion term in turbulent flows:

$$
\Gamma=\frac{\mu_{e f f}}{\sigma_{S C}},
$$

where $\mu_{\text {eff }}$ is the turbulent viscosity and $\sigma_{S C}$ is the Schmidt number. In the simulation it has a constant value of 0.7 .

Assuming steady state condition eqn (9) can be simplified as:

$$
\nabla \cdot\left(\rho \vec{v} \varphi-\frac{\mu_{e f f}}{\sigma_{S C}} \nabla \varphi\right)=1
$$

The boundary condition for eqn (11) is a zero value at the inlet and a zero gradient at the outflow boundary (Chanteloup and Mirade [6]).

For describing the different phases' behaviour Fluent Mixture model [10] was used. Continuity equation takes into account a mass averaged velocity, momentum equation sums up the individual momentum for all phases and a volume fraction as a scalar variable is introduced which can be calculated by dividing the secondary phase volume in respect with the total volume. Mixture model uses a so-called slip velocity, which is the velocity difference between the secondary and primary phase (Manninen et al. [13]).

The above mentioned equations - eqns. (1), (2), (4), (5), (11) and multiphase model - should be discretized and solved on a predefined mesh. For that second order discretisation scheme was used on all equations to reduce numerical diffusion and for the pressure-velocity coupling, the SIMPLE (Semi-Implicit Method for Pressure Linked Equations) scheme was required. 


\section{Results and discussion}

Study site was a small municipal wastewater treatment plant about 2000 PE in Sarisap, Hungary. The wastewater tank consists of two parts, one is the aeration tank with cylindrical form and a cone shaped settling tank as the figure 1 shows. The applied mesh is built with tetrahedral elements with approx. 550000 cell number and 107000 node number. Size function was used where significant gradients in flow field variables may occur, at the inflow, outflow and at the inner pipes. Boundary condition was a constant volume flux $\left(185 \mathrm{~m}^{3} / \mathrm{d}\right.$ due to the $85 \%$ cumulative frequency of the inflow water) at the inlet, an atmospherical pressure at the outlet. In the aeration tank air source term is built in the model to assume the effect of the aeration on the mixing. At the surface was the outflow of the air due to a negative source term to satisfy the continuity.
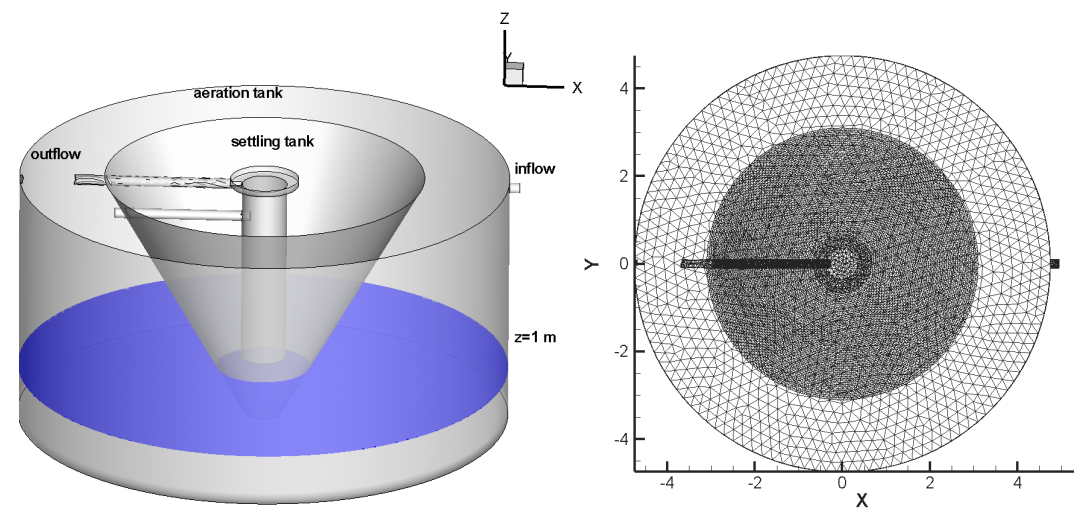

Figure 1: Aeration and settling tank geometry 3D Cartesian coordinates (left) and applied mesh 2D Cartesian coordinates (right).

As the solution converged, tracer was injected to the tank, replacing the original fluid and the outflow volume fraction of the tracer was detected. In the field study $\mathrm{LiCl}$ was discharged, which concentration was measured in the effluent water during five days. According to the literature (Buttz and Daigger [5]) five days the 95 percent of the $\mathrm{LiCl}$ added must be measured in the samples. The measured data can be transformed to a variable, which can be compared to the numerical results.

$$
\begin{aligned}
& E(t)=\frac{C(t)}{\int_{0}^{\infty} C(t) d t} \\
& F(t)=\int_{0}^{t} E(t) d t
\end{aligned}
$$


$E(t)$ is the normalised concentration response function and $F(t)$ is the cumulative response function, which comes from the numerical model. Figure 2 shows the measured and calculated data (Karches and Melicz [11]).

From the $F(t)$ the average residence time is:

$$
\bar{t}=\int_{0}^{\infty}(1-F(t)) d t
$$

Solving eqn (14), the average residence time from the measured data is around $40 \mathrm{~h}$, from the numerically calculated data it is $45,3 \mathrm{~h}$. In a previous study (Karches [11]) it is revealed that there was a hole between the aeration and the settling tank, therefore a small amount of fluid could escape faster than it was planned and diminish the residence time. The $\mathrm{V} / \mathrm{Q}$ ratio is $48,7 \mathrm{~h}$, which is close to the model result.



Figure 2: $\quad$ RTD study measured and calculated data which represents the mass fraction of the tracer at the outlet boundary.

When the flow field is given, LMA calculation (solving the eqn. 11) can be made separately. LMA predicts the average time which is needed to reach that point in the wastewater tank. Figures 3 and 4 show the distribution of the local fluid age at different sections. It can be seen that there are several zones where the fluid age is much higher than in the other zones. Because of the high fluid age the dissolved oxygen level decreases, anaerobic zones could appear in the aeration tank, which has a direct negative effect on the wastewater effluent quality. If a particle reaches the high fluid age zone, remains there for a long time, but not forever due to the turbulence. In the settling tank, because of the bad design, the fluid uses only a small volume, the residence time is not sufficient for the proper settling and the fact was confirmed by the LMA calculation. Another interesting fact is that aeration does not cease the deadzones, probably because of the low intensity of the air inflow. 
While RTD calculation predicts the occurrence of dead-zones, LMA shows the location, spatial dimensions of them. The problem of the dead-zone could be solved if the flow direction would not be the normal direction of the inlet boundary, but under an angle e.g. $60^{\circ}$. The settling problem could be solved if the outflow would be at the margin of the settling cone, because the particles path would be longer and there will be more time for the separation.

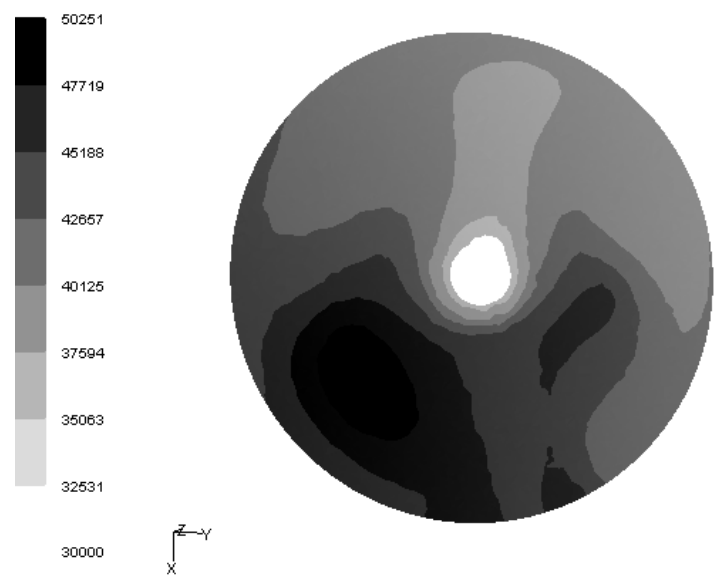

Figure 3: Local mean age of the fluid at $z=1 \mathrm{~m}$ plane. The dark colour represents the high water age. Scale is in second.

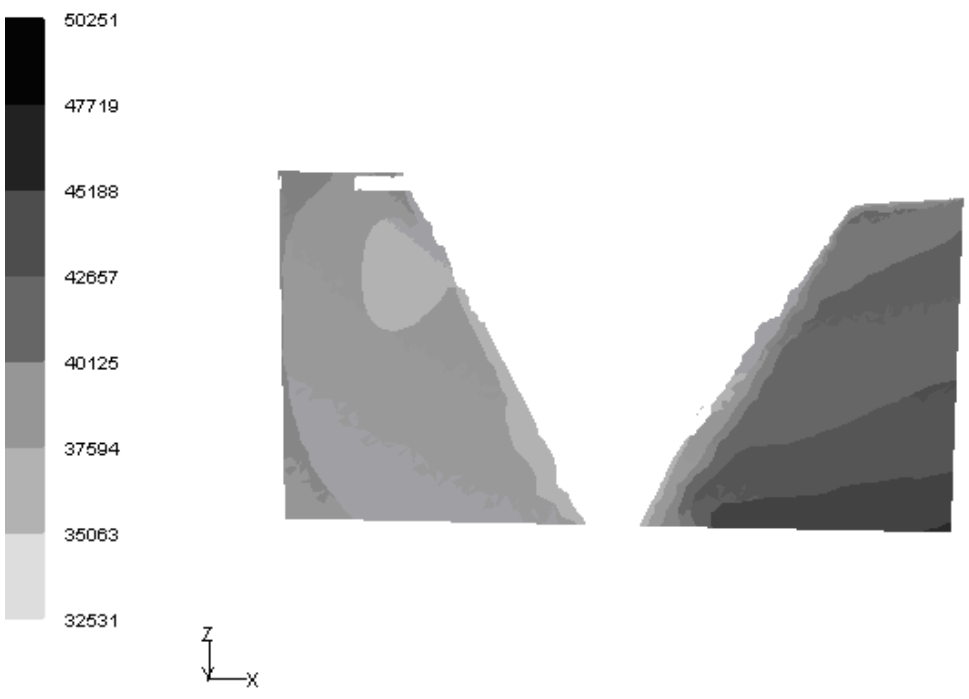

Figure 4: Local mean age of the fluid at XZ plane. The scale is in second. 


\section{Conclusion}

This study focused on the hydraulic aspects of a wastewater tanks. With the help of CFD the turbulence flow dynamics could have been predicted in a small wastewater treatment plant. Multiphase modelling was required on describing the aeration and carry out the RTD study with which could predict the average residence time in the wastewater tank and have impressions whether there are short-circuits and dead-zones. CFD flow field evaluation using velocity vectors, streamline imaging helped us to find location of the hydraulic phenomena but these effects on the residence time was not calculated. Local mean age of fluid particle is the link between the hydraulics and the biological processes. We find zones in the aeration tank, where the fluid's age was extreme high which could cause high ammonium concentration in the effluent. In the settling tank the residence time was not enough for the sedimentation process which results high suspended solid concentration in the effluent. With numerical simulation not only the problem detection is possible, but extensive knowledge of the fluid flow and residence time could help us to understand better the biological processes, too.

\section{Acknowledgements}

This work is connected to the scientific program of the "Development of qualityoriented and harmonized R+D+I strategy and functional model at BME" project. This project is supported by the New Hungary Development Plan (Project ID: TÁMOP-4.2.1/B-09/1/KMR-2010-0002

\section{References}

[1] ATV-DVWK Arbeitsblatt-A-131. GFA, Hennef, 2000.

[2] Bartrand, T.A., Farouk, B., Greene D.J., Haas C.N., Liberti L., Notarnicola M. and Santoro D., Use of CFD for wastewater disinfection process analysis: E.coli inactivation with peroxyacetic acid (PAA). International Journal of Chemical Reactor Engineering Vol. 3:A46, 2005.

[3] Bratchley E., Do-Quang Z., Janex M.L., Laine J.M. and Savoye P., Impact of water quality and reactor hydrodynamics on wastewater disinfection by UV, use of CFD modeling for performance optimization. Water Science and Technology Vol. 38 No. 6 pp. 71-78, 1998.

[4] Brouckaert C.J. and Buckley C.A., The use of Computational Fluid Dynamics for Improving the design and operation of water and wastewater treatment plants. Water Science and Technology Vol. 40 No. 4-5 pp. 81-90, 1999.

[5] Buttz J.A. and Daigger G.T. Upgrading wastewater treatment plants. Technomic Pub Co Vol 2. pp. 13-53, 1998.

[6] Chanteloup V. and Mirade P-S. Computational fluid dynamics (CFD) modelling of local mean age of air distribution in forced-ventilation food plants. Journal of Food Engineering Vol. 90 pp. 90-103, 2009. 
[7] Gentric C., Leclerc J.P., Moullec Y.L. and Potier O. Flow field and residence time distribution simulation of a cross-flow gas-liquid wastewater treatment reactor using CFD. Chemical Engineering Science Vol. 63 pp. 2436-2449, 2008.

[8] Greenfield P.F., Howes T., Johns M.R., Keller J. and Wood M.G. Computational fluid dynamic modelling of wastewater ponds to improve design. Water Science and Technology Vol. 31 No. 12 pp. 111-118, 1995.

[9] Foias C., Manley O. Rosa R. and Temam R. (2001) Navier-Stokes equations and turbulence Cambridge Books Online pp. 1-3.

[10] Fluent 6.3 Users Guide. Ansys Inc., USA

[11] Karches T. and Melicz Z., Problem detection in a small wastewater treatment tank with the tool of computational fluid dynamics. 9th international Congress on Advances in Civil Engineering.KTU, Trabzon, Turkey, 27-30 Sept. 2010.

[12] Launder B.E. and Spalding D.B. The numerical computation of turbulent flow. Computer methods in applied mechanics and Engineering, Vol. 3 Issue 2. pp. 269-289, 1974.

[13] Manninen M., Taivassalo V. and Kallio S. On the mixture model for multiphase flow. VTT Publications 288, Technical Research Centre of Finland, 1996.

[14] Saul J. A. and Stovin R.V. A computational fluid dynamics (CFD) particle tracking approach to efficiency prediction. Water Science and Technology Vol.37. Issue 1 pp. 285-293, 1998.

[15] Simons MW., Waters J. R. and White P. R. S., Local mean age of air: predictive techniques compared. Building Services Engineering Research and Technology Vol. 20. No. 4211-218, 1999.

[16] Ta CT, Beckley J., and Eades A. A multiphase CFD model of DAF process. Water Science and Technology Vol. 43 No 8 pp. 153-157, 2001.

[17] Yang Y., Wu Y., Yang X., Zhang K. and Yang J. Flow field prediction in full-scale Carrousel oxidation ditch by using computational fluid dynamics Water Science and Technology Vol. 62 No 2 pp. 256-265, 2010. 